\title{
An Algorithm for Imaging Isochrones of Ventricular Activation on Patient-Specific Epicardial Surface
}

\author{
Shijie Zhou ${ }^{1}$, John L Sapp ${ }^{2}$, Amir AbdelWahab ${ }^{2}$, B Milan Horáček ${ }^{1}$ \\ ${ }^{1}$ School of Biomedical Engineering, Dalhousie University, Halifax, NS, Canada \\ ${ }^{2}$ Division of Cardiology, Department of Medicine, Dalhousie University, Halifax, NS, Canada
}

\begin{abstract}
Electrocardiographic imaging has been shown to provide useful information for pre-procedure planning of catheter-ablation procedures. The methodology involves reconstruction of unipolar electrograms (EGMs) and isochronal maps on the epicardial surface from noninvasively acquired body-surface potentials. We have developed an algorithm for evaluating global myocardial activation times. First, the cross-correlation method determines the delay in local activation times among pairs of neighboring nodes. Next, a sparse linear system is constructed from known activation delays of neighboring nodes. To solve this system, we use a sparse Bayesian learning method to calculate the global myocardial activation times. The aim of this study was to assess the proposed method in both structurally normal and scarred ventricular myocardium. Isochronal maps of calculated activation times were compared with local activation times (LATs) derived from directly-measured epicardial EGMs obtained by electroanatomic contact mapping, for pacing delivered by an implantable cardioverter defibrillator (ICD) at the endocardial right-ventricular $(R V)$ apex, and for catheter pacing at $R V$ epicardial site. We found that even in the presence of infarct scar, isochronal maps calculated by the proposed method correlated closely with known LATs exported from an electroanatomic mapping system.
\end{abstract}

\section{Introduction}

Clinical electrophysiological (EP) studies are routinely used to investigate abnormal propagation patterns by techniques of activation mapping [1]. The isochronal activation map visualizes propagation of the activation wave front in the myocardium and it provides a summary of an entire activation sequence. Over the past decade, a noninvasive modality of electrocardiographic imaging (ECGI) has demonstrated its ability to reconstruct unipolar electrograms (EGMs) and isochronal maps on the epicardial surface from measured body-surface potential distribu- tions [2]. There are two most-frequently used methods for calculating activation times by means of the ECGI.

In a standard computation, the local activation time (LAT) is defined as the time when the activation wave front passes beneath the electrode, which is usually detected either as the maximum negative slope of the electrical deflection for computed unipolar EGM for that location, or as the maximum signal amplitude in bipolar recordings [3]. Alternatively, a nonlinear temporal model based on a priori restriction of transmembrane potential waveforms can be adopted on the heart surface [4]; this technique is referred to as activation-time imaging. However, the latter approach-with its a priori restrictions-does not accurately describe the full temporal behavior of the heartsurface potentials and it can be especially problematic in the cases of acute ischemia or prior myocardial infarction. Moreover, the activation-time imaging requires strict physiological constraints based on the assumption of uniformdouble-layer (UDL) wave front [5].

Our study presents a new approach for constructing isochronal activation maps, based on propagation delays at neighboring locations [6]. First, the generalized crosscorrelation method [7] is used to determine delays in activation times among pairs of neighboring locations. Next, a sparse linear system is constructed by using known activation delays as inputs. To solve this system, we use a sparse Bayesian learning method to calculate global myocardial activation times. This new method of detecting activation times was assessed by correlation with known activation maps derived from directly measured EGMs collected invasively through an electroanatomic system (Carto ${ }^{\mathbb{R}}$, Biosense Webster, Inc., Irvine, CA, USA).

\section{Methods}

\subsection{Study population}

A single patient with recurrent scar-related ventricular tachycardia (VT) undergoing epicardial catheter ablation had 120-lead body-surface potential mapping (BSPM) per- 
formed. The patient was one of 4 patients used in a previous study [2]. The study protocol was approved by the Institutional Research Ethics Board of Nova Scotia Health Authority, the patient gave written informed consent.

\subsection{Inverse solution}

A linear model relating epicardial surface potentials to measured body-surface potentials through a transfer matrix $A$ was calculated by using the boundary element method (BEM) [8]. The epicardial potential source model was used to calculate the forward problem of electrocardiographic modeling [9], which derives the transfer matrix $A$ by solving the boundary-integral formulation of Laplace's equation in the source-free volume conductor between the epicardial and torso surfaces. The $L_{1}$-norm regularization [10] was used to reconstruct electrograms (EGMs) that depict the variation of voltage as a function of time at a given point on the epicardial surface. We extracted the body-surface potential on the torso at each time instant and used it as the input for the inverse solution to recover a time sequence of potentials (EGMs); the regularization parameter was determined using the $L$-curve method [11].

\subsection{Global myocardial activation times}

Time delays calculated by generalized cross-correlation method [7] in the activation of two neighboring EGMs were used to construct a global myocardial activation map. After solving the cross-correlation for each couple of neighboring locations, the maximum (or minimum if the signals are negatively correlated) of the cross-correlation function indicates the instant of time at which the signals are best aligned, i.e., the time delay between the two signals is determined by the argument of the maximum, as in $\tau_{\text {delay }}=\arg \max ((f \star g)(t))$, where the $\star$ is the convolution operation for two continuous functions $f$ and $g$ based on time series, such as a couple of computed EGMs in our application.

Let us assume $N$ computed EGMs for which the activation map is known on the epicardial surface. For spatially local EGMs, an activation time delay between two nearby EGMs ( $i$ and $j$ ) is equal to time differential for the propagation wave front to travel from one $(i)$ to the other $(j)$ :

$$
T_{j}-T_{i}=\tau_{i j} \quad i, j=1, \ldots, N ; j>i .
$$

The above Eq. 1 implies a known activation relationships of two neighboring locations on the epicardial surface, which can be rewritten by a matrix form $R T=\tau$, where $T=\left[T_{1}, \ldots, T_{N}\right]^{\prime}$ is the vector of activation times on the epicardial surface, each row of the matrix $R$ with a sparse property corresponds to an equation for a given two neighboring locations, and the vector $\tau$ includes all pairs of EGMs' time delays $\left(\tau_{i j}, j>i\right)$. We used the Sparse Bayesian Learning [12] to solve the linear sparse system.

\subsection{Experimental studies}

We validated the proposed method of activation mapping estimated on Patient \#3 from our previous study [2] who had an implantable cardioverter defibrillator (ICD) device undergoing epicardial catheter ablation. During the ablation procedure, the reference (local activation times (LATs)) was measured when keeping the endocardial ICD device pacing located on the right ventricular (RV) apex. We also present a RV epicardial-pacing example where we used the proposed method to generate global myocardial activation-time maps to depict the propagation waveforms.

\section{Results}

Fig. 1 (panels E-H) shows the measured LATs obtained from Carto ${ }^{\circledR}$ electroanatomic mapping system by using contact mapping. The estimated isochronal maps (Fig. 1, panels I-L) obtained by the proposed method show a qualitatively similar spread of activation with the measured isochronal maps (Fig. 1, panels E-H), and feature several characteristics when combining the measured isochronal maps with bipolar substrate maps to analyze: The earliest activation was captured on the RV apical region indicated by red color (panels $\mathrm{J}$ and $\mathrm{K}$ ); and then the activation wave spreads over the RV lateral wall, RV anterior wall and RV inferior wall in approximately $100 \mathrm{~ms}, 200$ $\mathrm{ms}$ and $180 \mathrm{~ms}$, respectively, to the latest activation of the LV basal lateral wall at around $220 \mathrm{~ms}$ colored by dark blue (panel L). Moreover, a region with diminished signal amplitude on contact electrogram (panel A indicated by a black circle) is contiguous with basolateral LV epicardial scar, and in continuity with the interventricular groove, at a site of expected epicardial adipose tissue, is associated with abnormal propagation (indicated by a black circle) as shown in the basal anterior view of panel I. We cannot be certain whether this represents actual extension of scar or fat tissue. In Fig. 1, the estimated isochronal maps of panels $\mathrm{K}$ and $\mathrm{L}$ suggest abnormal propagation through the LV inferior apical areas (identified by a black circle on panel K) and slow propagation through LV basal anterolateral wall (panel $\mathrm{L}$ indicated by a yellow circle), respectively, correlating with diminished bipolar contact electrogram amplitude, suggesting scar (marked by a black circle on panel C, and a yellow circle on panel D). Panel D depicts a large scar on the LV lateral wall extending to the apical region (identified by a black circle), associated with abnormal propagation through the same location of the estimated isochronal map of panel L (identified by a black circle). In addition, the estimated isochronal map of panel $\mathrm{J}$ illustrating normal propagation waveforms on the RV lat- 

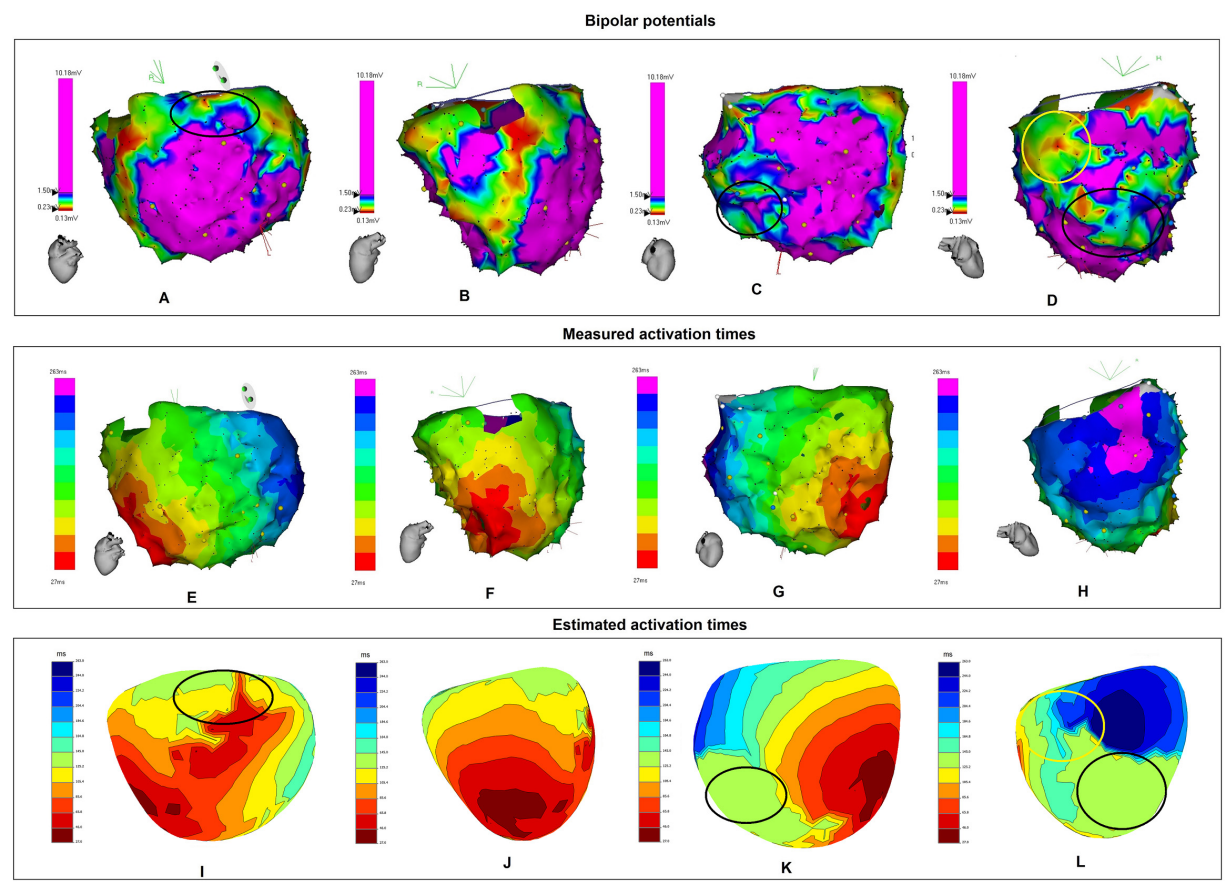

Figure 1. The first row (panels A-D) shows Carto ${ }^{\circledR}$ epicardial substrate maps based on bipolar potentials with areas featuring bipolar signal amplitude $\geq 1.5 \mathrm{mV}$ in purple. The second row (panels E-H) depicts the LATs as measured by Carto ${ }^{R}$ system; earliest activation is indicated by red. The third row (panels I-L) shows global myocardial activation times estimated by the proposed method; red and dark blue represent the earliest and the latest activation, respectively.

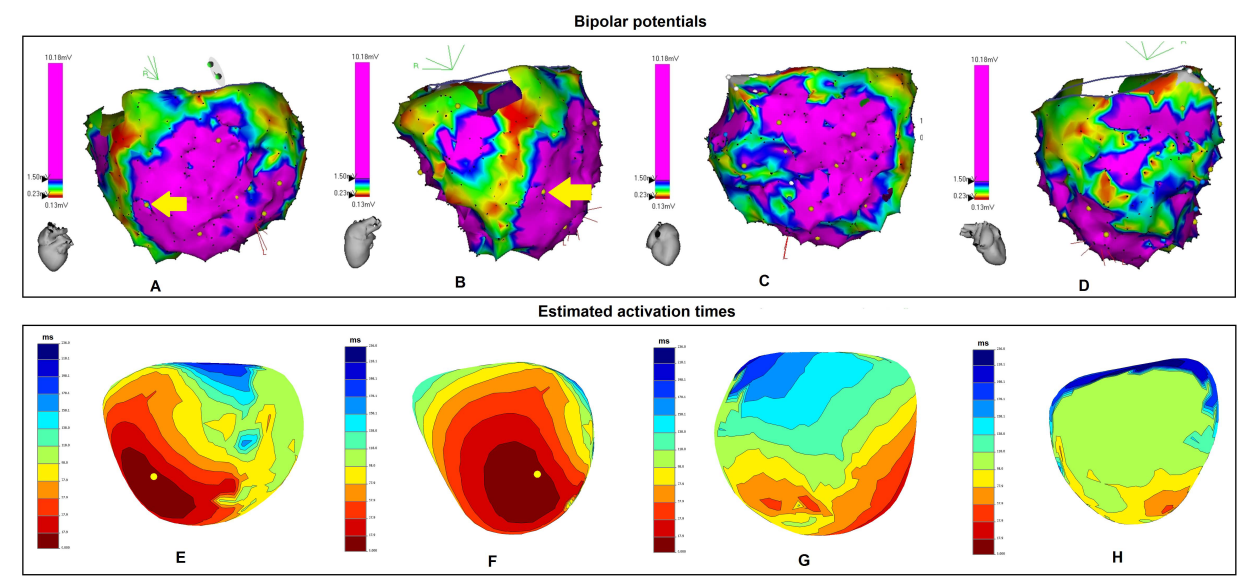

Figure 2. The first row (panels A-D) shows Carto ${ }^{\circledR}$ epicardial substrate maps based on bipolar potentials; the second row (panels E-H) shows global myocardial activation-time maps estimated by the proposed method. The yellow arrow indicates an epicardial pacing site as the activation origin on electroanatomic maps; the yellow dot indicates where the epicardial pacing site projects onto the estimated global myocardial activation-time maps; red and dark blue represent the earliest and the latest activation, respectively.

eral and basal anterolateral wall suggest that the low bipolar contact electrogam potentials $(<1.5 \mathrm{mV})$ on the electroanatomic map could be caused by the epicardial fat in the interventricular groove (Fig. 1, panel B).
Fig. 2 (panels E-H) illustrate the global myocardial activation maps constructed from a RV epicardial pacing site located on mid anterior RV (more close apical region) indicated on the bipolar substrate maps by a yellow arrow 
on panels A and B. The estimated activation times were able to capture earliest activation areas and resulted in a constructed propagation wave front; and the propagation waveforms show several features on the epicardial surface: A large scar region with lowest bipolar potentials (panel A) was located on the LV basal anterolateral wall, causing delayed propagation (indicated by the green color part) as shown in the LV basal anterolateral view of panel E. The estimated isochronal map (panel $\mathrm{G}$ ) shows the activation wave spreads over the RV inferior wall (healthy tissue) with normal propagation, and through the scarred LV inferolateral wall (near apical region) with delayed propagation. Further, the area with abnormal activation was located in the lateral region of the $\mathrm{LV}$, which has a large scar and scar margin on panel D. Finally, the estimated isochronal map of panel $\mathrm{F}$ also featured the normal propagation on the RV lateral wall, which further confirms our previous impression that RV epicardial fat is responsible for the large area of reduced electrogram amplitude seen in panel B.

\section{Conclusion}

The principal goal of this study was to present an approach for assessing the global myocardial activation times at the sites of computed EGMs. The proposed method, based on the cross-correlation method to calculate the time delays from the heart with diseased tissue, is more accurate than the standard computation (steepest down-slope method), achieving a smaller standard deviation. Inversesolution isochrones yielded by this algorithm correlated closely with known activation maps obtained by contact mapping during an ICD endocardial pacing at the RV. We also found that the proposed method may reflect the presence of the scar that approximately correlated with known electroanatomic potential maps exported from an electroanatomic mapping system. The promising results demonstrate that the proposed method can non-invasively estimate the global myocardial activation times and accurately capture abnormal propagation patterns for a patient with diseased myocardium. Further validation in a largerscale study is needed to confirm.

\section{Acknowledgements}

This work was supported in part by the Natural Sciences and Engineering Research Council of Canada, the Nova Scotia Health Research Foundation, the Nova Scotia Research and Innovation Graduate Scholarship, the Heart $\&$ Stroke Foundation of Nova Scotia, and the Cardiac Arrhythmia Network of Canada (CANet).

\section{References}

[1] Man KC, Knight B, Tse HF, et al. Radiofrequency catheter ablation of inappropriate sinus tachycardia guided by activation mapping. J. Am. Coll. Cardiol., 35(2):451-457, 2000.

[2] Sapp JL, Dawoud F, Clements JC, Horáček BM. "Inverse solution mapping of epicardial potentials: quantitative comparison with epicardial contact mapping," Circ Arrhythm Electrophysiol, 5(5):1001-9, 2012.

[3] Spach MS, Miller WT, Miller-Jones E, et al. Extracellular potentials related to intracellular action potentials during impulse conduction in anisotropic canine cardiac muscle. Circ. Res., 45(2):188-204, 1979.

[4] van Dam PM, Oostendorp TF, Linnenbank AC, van Oosterom A. Non-invasive imaging of cardiac activation and recovery. Ann Biomed Eng, 37(9):1739-1756, 2009.

[5] Taccardi B, Punske BB, Lux RL, et al. Useful lessons from body surface mapping. J. Cardiovasc. Electrophysiol., 9(7):773-786, 1998.

[6] Dubois R, Labarthe S, Coudiere Y, et al. Global and directional activation maps for cardiac mapping in electrophysiology. In 2012 Computing in Cardiology, pages 349-352, 2012.

[7] Azaria M, Hertz D. Time delay estimation by generalized cross correlation methods. IEEE Trans Acoustics, Speech, and Signal Processing, 32(2):280-285, 1984.

[8] Brebbia CA, Telles JCF, Wrobel LC. Boundary Element Techniques: Theory and Applications in Engineering, pages 64-70. Berlin: Springer-Verlag, 1984.

[9] Horáček BM, Clements JC. The inverse problem of electrocardiography: a solution in terms of single- and doublelayer sources of the epicardial surface. Math Biosci, 144(2):119-154, 1997.

[10] Ghosh S, Rudy Y. Application of L1-norm regularization to epicardial potential solution of the inverse electrocardiography problem. Ann Biomed Eng, 37(5):902-12, 2009.

[11] Hansen PC, O'Leary DP. The use of the $L$-curve in the regularization of discrete ill-posed problems. SIAM J Sci Comp, 14(6):1487-1503, 1993.

[12] Wipf DP, Rao BD. Sparse Bayesian learning for basis selection. IEEE Transactions on Signal Processing, 52(8):2153-2164, 2004.

Address for correspondence:

Shijie Zhou (Shijie.Zhou@dal.ca)

School of Biomedical Engineering / Dalhousie University

Halifax, NS / Canada 